\title{
A parallel Jacobian-free Newton-Krylov solver for a coupled sea ice-ocean model
}

\author{
Martin Losch ${ }^{\mathrm{a}, *}$, Annika Fuchs ${ }^{\mathrm{a}}$, Jean-François Lemieux ${ }^{\mathrm{b}}$, Anna Vanselow ${ }^{\mathrm{c}}$ \\ ${ }^{a}$ Alfred-Wegener-Institut, Helmholtz Zentrum für Polar- und Meeresforschung, Postfach \\ 120161, 27515 Bremerhaven, Germany \\ ${ }^{b}$ Recherche en Prévision Numérique environnementale/Environnement Canada, 2121 \\ route Transcanadienne, Dorval, Qc, Canada H9P 1 J3 \\ ${ }^{c}$ Universität Oldenburg, Ammerländer Heerstr. 114-118, 26129 Oldenburg
}

\begin{abstract}
The most common representation of sea ice dynamics in climate models assumes that sea ice is a quasi-continuous non-normal fluid with a viscousplastic rheology. This rheology leads to non-linear sea ice momentum equations that are notoriously difficult to solve. Recently a Jacobian-free NewtonKrylov (JFNK) solver was shown to solve the equations accurately at moderate costs. This solver is extended for massive parallel architectures and vector computers and implemented in a coupled sea ice-ocean general circulation model for climate studies. Numerical performance is discussed along with numerical difficulties in realistic applications with up to 1920 CPUs. The parallel JFNK-solver's scalability competes with traditional solvers although the collective communication overhead starts to show a little earlier. When accuracy of the solution is required (i.e. reduction of the residual norm of the momentum equations of more that one or two orders of magnitude) the JFNK-solver is unrivalled in efficiency. The new implementation opens up the opportunity to explore physical mechanisms in the context of large scale sea ice models and climate models and to clearly differentiate these physical effects from numerical artifacts.
\end{abstract}

Keywords: sea ice dynamics, numerical sea ice modeling, Jacobian-free Newton-Krylov solver, preconditioning, parallel implementation, vector implementation

\footnotetext{
*corresponding author

Email address: Martin.Losch@awi.de (Martin Losch)
} 


\section{Introduction}

The polar oceans are geographically small compared to the world ocean, but still they are a very influential part of Earth's climate. Sea ice is an important component of the polar oceans. It acts as an insulator of heat and surface stress and without it atmospheric temperatures and hence flow patterns would be entirely different than today. Consequently, predicting future climate states or hindcasting previous ones requires accurate sea ice models $[1,2]$. The motion of sea ice from formation sites to melting sites determines many aspects of the sea ice distribution and virtually all stateof-the-art sea ice models explicitly include a dynamics module.

Unfortunately, climate sea ice models necessarily contain many approximations that preclude the accurate description of sea ice dynamics. First of all, sea ice is usually treated as a quasi-continuous non-Newtonian fluid with a viscous-plastic rheology [3]. The assumption of quasi-continuity may be appropriate at low resolution but at high resolution (i.e. with a grid spacing on the order of kilometers) the scale of individual floes is reached and entirely new approaches may be necessary $[4,5,6]$. If continuity is acceptable (as in climate models with grid resolutions of tens of kilometers), the details of the rheology require attention $[7,8,6]$. Lemieux and Tremblay [9] and Lemieux et al. [10] demonstrated that the implicit numerical solvers that are used in climate sea ice models do not yield accurate solutions. These Picard solvers suffer from poor convergence rates so that iterating them to convergence is prohibitive [10]. Instead, a typical iterative process is terminated after a few (order two to ten) non-linear (or outer loop, OL) steps assuming falsely that the solution is sufficiently accurate $[11,9]$. Without sufficient solution accuracy, the physical effects, that is, details of the rheology and improvements by new rheologies cannot be separated from numerical errors $[12,13]$. Explicit methods may not converge at all [10].

Lemieux et al. [14] implemented a non-linear Jacobian-free Newton-Krylov (JFNK) solver in a serial sea model and demonstrated that this solver can give very accurate solutions compared to traditional solvers with comparatively low cost [10]. Here, we introduce and present the first JFNK-based sea ice model coupled to a general circulation model for parallel and vector computers. For this purpose, the JFNK solver was parallelized and vectorized. The parallelization required introducing a restricted additive Schwarz 
method (RASM) [15] into the iterative preconditioning technique (line successive relaxation, LSR) and the parallelization of the linear solver; the vector code also required revisiting the convergence of the iterative preconditioning method (LSR). The JFNK solver is matrix free, that is, only the product of the Jacobian times a vector is required. The accuracy of this operation is studied. Exact solutions with a tangent-linear model are compared to more efficient finite-difference approaches.

Previous parallel JFNK solutions addressed compressible flow [16] or radiative transfer problems [17]. The sea ice momentum equations stand apart because the poor condition number of the coefficient matrix makes the system of equations very difficult to solve [9]. The coefficients vary over many orders of magnitude because they depend exponentially on the partial ice cover within a grid cell (maybe comparable to Richards' equations for fluid flow in partially saturated porous media [18]) and are a complicated function (inverse of a square root of a quadratic expression) of the horizontal derivatives of the solution, that is, the ice drift velocities. These are very different in convergent motion where sea ice can resist large compressive stress and in divergent motion where sea ice has very little tensile strength. As a consequence, a successful JFNK solver for sea ice momentum equations requires great care, and many details affect the convergence. For example, in contrast to Godoy and Liu [17], we never observed convergence in realistic simulations without sufficient preconditioning.

The paper is organized as follows. In Section 2 we review the sea ice momentum equations and the JFNK-solver; we describe the issues that needed addressing and the experiments that are used to illustrate the performance of the JFNK-solver. Section 3 discusses the results of the experiments and conclusions are drawn in Section 4.

\section{Model and Methods}

For all computations we use the Massachusetts Institute of Technology general circulation model $(\mathrm{MITgcm})$ code $[19,20]$. This code is a general purpose, finite-volume algorithm on regular orthogonal curvilinear grids that solves the Boussinesq and hydrostatic form of the Navier-Stokes equations for an incompressible fluid with parameterizations appropriate for oceanic or atmospheric flow. (Relaxing the Boussinesq and hydrostatic approximations is possible, but not relevant here.) For on-line documentation of the general algorithm and access to the code, see http://mitgcm.org. The MITgcm 
contains a sea ice module whose dynamic part is based on Hibler's [3] original work; the code has been rewritten for an Arakawa C-grid and extended to include different solution techniques and rheologies on curvilinear grids [12]. The sea ice module serves as the basis for implementation of the JFNK solver.

\subsection{Model Equations and Solution Techniques}

The sea ice module of the MITgcm is described in Losch et al. [12]. Here we reproduce a few relevant aspects. The momentum equations are

$$
m \frac{D \mathbf{u}}{D t}=-m f \mathbf{k} \times \mathbf{u}+\boldsymbol{\tau}_{\text {air }}+\boldsymbol{\tau}_{\text {ocean }}-m \nabla \phi(0)+\mathbf{F},
$$

where $m$ is the combined mass of ice and snow per unit area; $\mathbf{u}=u \mathbf{i}+v \mathbf{j}$ is the ice velocity vector; $\mathbf{i}, \mathbf{j}$, and $\mathbf{k}$ are unit vectors in the $x-, y$-, and $z$ directions; $f$ is the Coriolis parameter; $\boldsymbol{\tau}_{\text {air }}$ and $\boldsymbol{\tau}_{\text {ocean }}$ are the atmosphere-ice and ice-ocean stresses; $\nabla \phi(0)$ is the gradient of the sea surface height times gravity; and $\mathbf{F}=\nabla \cdot \sigma$ is the divergence of the internal ice stress tensor $\sigma_{i j}$. Advection of sea-ice momentum is neglected. The ice velocities are used to advect ice compactness (concentration) $c$ and ice volume, expressed as cell averaged thickness $h c ; h$ is the ice thickness. The numerical advection scheme is a so-called 3rd-order direct-space-time method [21] with a flux limiter [22] to avoid unphysical over and undershoots. The remainder of the section focuses on solving (1).

For an isotropic system the stress tensor $\sigma_{i j}(i, j=1,2)$ can be related to the ice strain rate tensor

$$
\dot{\epsilon}_{i j}=\frac{1}{2}\left(\frac{\partial u_{i}}{\partial x_{j}}+\frac{\partial u_{j}}{\partial x_{i}}\right)
$$

and the ice pressure

$$
P=P^{*} c h e^{[-C \cdot(1-c)]}
$$

by a nonlinear viscous-plastic (VP) constitutive law $[3,11]$ :

$$
\sigma_{i j}=2 \eta \dot{\epsilon}_{i j}+[\zeta-\eta] \dot{\epsilon}_{k k} \delta_{i j}-\frac{P}{2} \delta_{i j} .
$$

The ice pressure $P$, a measure of ice strength, depends on both thickness $h$ and compactness (concentration) $c$; the remaining constants are set to 
$P^{*}=27500 \mathrm{~N} \mathrm{~m}^{-2}$ and $C=20$. We introduce the shear deformation $\dot{\epsilon}_{s}=$ $\sqrt{\left(\dot{\epsilon}_{11}-\dot{\epsilon}_{22}\right)^{2}+\dot{\epsilon}_{12}^{2}}$, the shear divergence $\dot{\epsilon}_{d}=\dot{\epsilon}_{11}+\dot{\epsilon}_{22}$, and the abbreviation $\Delta=\sqrt{\dot{\epsilon}_{d}^{2}+\dot{\epsilon}_{s}^{2} / e^{2}}$. The nonlinear bulk and shear viscosities $\zeta=P /(2 \Delta)$ and $\eta=\zeta / e^{2}$ are functions of ice strain rate invariants and ice strength such that the principal components of the stress lie on an elliptical yield curve with the ratio of major to minor axis $e=2$.

Substituting (2) into (1) yields equations in $u$ and $v$ that contain highly non-linear viscosity-like terms with spatially and temporally variable coefficients $\zeta$ and $\eta$; these terms dominate the momentum balance. $\Delta$ can be very small where ice is thick and rigid so that $\zeta$ and $\eta$ can span several orders of magnitude making the non-linear equations very difficult to solve, and some regularization is required. Following Lemieux et al. [10], the bulk viscosities are bounded smoothly from above by imposing a maximum $\zeta_{\max }=P /\left(2 \Delta^{*}\right)$, with $\Delta^{*}=2 \times 10^{-9} \mathrm{~s}^{-1}$ :

$$
\begin{aligned}
\zeta & =\zeta_{\max } \tanh \left(\frac{P}{2 \min \left(\Delta, \Delta_{\min }\right) \zeta_{\max }}\right) \\
& =\frac{P}{2 \Delta^{*}} \tanh \left(\frac{\Delta^{*}}{\min \left(\Delta, \Delta_{\min }\right)}\right)
\end{aligned}
$$

$\Delta_{\min }=10^{-20} \mathrm{~s}^{-1}$ is chosen to avoid divisions by zero. Alternatively, one could use a differentiable formula such as $\zeta=P /\left[2\left(\Delta+\Delta^{*}\right)\right]$, but in any case the problem remains poorly conditioned. After regularizing the viscosities, the pressure replacement method is used to compute the pressure as $2 \Delta \zeta$ [23]. For details of the spatial discretization, see Losch et al. [12]. For the following discussion, the temporal discretization is implicit in time following Lemieux et al. [10].

The discretized momentum equations can be written in matrix notation as

$$
\mathrm{A}(\mathrm{x}) \mathrm{x}=\mathrm{b}(\mathrm{x}) .
$$

The solution vector $\mathbf{x}$ consists of the two velocity components $u$ and $v$ that contain the velocity variables at all grid points and at one time level. In the sea ice component of the MITgcm, as in many sea ice models, Eq. (4) is solved with an iterative Picard solver: in the $k$-th iteration a linearized form $\mathbf{A}\left(\mathbf{x}^{k-1}\right) \mathbf{x}^{k}=\mathbf{b}\left(\mathbf{x}^{k-1}\right)$ is solved (in the case of the MITgcm it is an LSR-algorithm [11], but other methods may be more efficient [24]). Picard solvers converge slowly, but generally the iteration is terminated after only a few non-linear steps $[11,9]$ and the calculation continues with the next time 
level. Alternatively, the viscous-plastic constitutive law can be modified to elastic-viscous-plastic (EVP) to allow a completely explicit time stepping scheme [25]. These EVP-solvers are very popular because they are fast and efficient for massive parallel applications, but their convergence properties are under debate [10]. The EVP-solver in the MITgcm [12, 13] is extended to the modified EVP*-solver [10] for all EVP simulations.

The Newton method transforms minimizing the residual $\mathbf{F}(\mathbf{x})=\mathbf{A}(\mathbf{x}) \mathbf{x}-$ $\mathbf{b}(\mathbf{x})$ to finding the roots of a multivariate Taylor expansion of the residual $\mathbf{F}$ around the previous $(k-1)$ estimate $\mathbf{x}^{k-1}$ :

$$
\mathbf{F}\left(\mathbf{x}^{k-1}+\delta \mathbf{x}^{k}\right)=\mathbf{F}\left(\mathbf{x}^{k-1}\right)+\mathbf{F}^{\prime}\left(\mathbf{x}^{k-1}\right) \delta \mathbf{x}^{k}
$$

with the Jacobian $\mathbf{J} \equiv \mathbf{F}^{\prime}$. The root $\mathbf{F}\left(\mathbf{x}^{k-1}+\delta \mathbf{x}^{k}\right)=0$ is found by solving

$$
\mathbf{J}\left(\mathbf{x}^{k-1}\right) \delta \mathbf{x}^{k}=-\mathbf{F}\left(\mathbf{x}^{k-1}\right)
$$

for $\delta \mathbf{x}^{k}$. The next $\left(k\right.$-th) estimate is given by $\mathbf{x}^{k}=\mathbf{x}^{k-1}+a \delta \mathbf{x}^{k}$. In order to avoid overshoots the factor $a$ is iteratively reduced in a line search $(a=$ $\left.1, \frac{1}{2}, \frac{1}{4}, \frac{1}{8}, \ldots\right)$ until $\left\|\mathbf{F}\left(\mathbf{x}^{k}\right)\right\|<\left\|\mathbf{F}\left(\mathbf{x}^{k-1}\right)\right\|$, where $\|\cdot\|=\int \cdot d x^{2}$ is the $L_{2}$-norm. In practice, the line search is stopped at $a=\frac{1}{8}$.

Forming the Jacobian $\mathbf{J}$ explicitly is often avoided as "too error prone and time consuming" [26]. Instead, Krylov methods only require the action of $\mathbf{J}$ on an arbitrary vector $\mathbf{w}$ and hence allow a matrix free algorithm for solving Eq. (6) [26]. The action of $\mathbf{J}$ can be approximated by a first-order Taylor series expansion [26]:

$$
\mathbf{J}\left(\mathbf{x}^{k-1}\right) \mathbf{w} \approx \frac{\mathbf{F}\left(\mathbf{x}^{k-1}+\epsilon \mathbf{W}\right)-\mathbf{F}\left(\mathbf{x}^{k-1}\right)}{\epsilon}
$$

or computed exactly with the help of automatic differentiation (AD) tools [16]. Besides the finite-difference approach we use TAF (http://www . fastopt . de) or TAMC [27] to obtain the action of $\mathbf{J}$ on a vector. The MITgcm is tailored to be used with these tools [28] so that obtaining the required code with the help of a tangent linear model is straightforward.

We use the Flexible Generalized Minimum RESidual method (FGMRES, [29]) with right-hand side preconditioning to solve Eq. (6) iteratively starting from a first guess of $\delta \mathbf{x}_{0}^{k}=0$. For the preconditioning matrix $\mathbf{P}$ we choose a simplified form of the system matrix $\mathbf{A}\left(\mathbf{x}^{k-1}\right)[14]$ where $\mathbf{x}^{k-1}$ is the estimate of the previous Newton step $k-1$. The transformed equation (6) becomes

$$
\mathbf{J}\left(\mathbf{x}^{k-1}\right) \mathbf{P}^{-1} \delta \mathbf{z}=-\mathbf{F}\left(\mathbf{x}^{k-1}\right), \quad \text { with } \quad \delta \mathbf{z}=\mathbf{P} \delta \mathbf{x}^{k} .
$$


The Krylov method iteratively improves the approximate solution to (8) in subspace $\left(\mathbf{r}_{0}, \mathbf{J P}^{-1} \mathbf{r}_{0},\left(\mathbf{J P}^{-1}\right)^{2} \mathbf{r}_{0}, \ldots,\left(\mathbf{J P}^{-1}\right)^{m} \mathbf{r}_{0}\right)$ with increasing $m ; \mathbf{r}_{0}=$ $-\mathbf{F}\left(\mathbf{x}^{k-1}\right)-\mathbf{J}\left(\mathbf{x}^{k-1}\right) \delta \mathbf{x}_{0}^{k}$ is the initial residual of $(6) ; \mathbf{r}_{0}=-\mathbf{F}\left(\mathbf{x}^{k-1}\right)$ with the first guess $\delta \mathbf{x}_{0}^{k}=0$. We allow a Krylov-subspace of dimension $m=50$ and we do not use restarts. The preconditioning operation involves applying $\mathbf{P}^{-1}$ to the basis vectors $\mathbf{v}_{0}, \mathbf{v}_{1}, \mathbf{v}_{2}, \ldots, \mathbf{v}_{m}$ of the Krylov subspace. This operation is approximated by solving the linear system $\mathbf{P} \mathbf{w}=\mathbf{v}_{i}$. Because $\mathbf{P} \approx \mathbf{A}\left(\mathbf{x}^{k-1}\right)$, we can use the LSR-algorithm [11] already implemented in the Picard solver. Each preconditioning operation uses a fixed number of 10 LSR-iterations avoiding any termination criterion. More details can be found in [14].

The non-linear Newton iteration is terminated when the $L_{2}$-norm of the residual is reduced by $\gamma_{\mathrm{nl}}$ with respect to the initial norm: $\left\|\mathbf{F}\left(\mathbf{x}^{k}\right)\right\|<$ $\gamma_{\mathrm{nl}}\left\|\mathbf{F}\left(\mathbf{x}^{0}\right)\right\|$. Within a non-linear iteration, the linear FGMRES solver is terminated when the residual is smaller than $\gamma_{k}\left\|\mathbf{F}\left(\mathbf{x}^{k-1}\right)\right\|$ where $\gamma_{k}$ is determined by

$$
\gamma_{k}= \begin{cases}\gamma_{0} & \text { for }\left\|\mathbf{F}\left(\mathbf{x}^{k-1}\right)\right\| \geq r \\ \max \left(\gamma_{\min }, \frac{\left\|\mathbf{F}\left(\mathbf{x}^{k-1}\right)\right\|}{\left\|\mathbf{F}\left(\mathbf{x}^{k-2}\right)\right\|}\right) & \text { for }\left\|\mathbf{F}\left(\mathbf{x}^{k-1}\right)\right\|<r\end{cases}
$$

so that the linear tolerance parameter $\gamma_{k}$ decreases with the non-linear Newton step as the non-linear solution is approached. This inexact Newton method is generally more robust and computationally more efficient than exact methods $[30,26]$. We choose $\gamma_{0}=0.99, \gamma_{\min }=0.1$, and $r=\frac{1}{2}\left\|\mathbf{F}\left(\mathbf{x}^{0}\right)\right\|$; we allow up to 100 Newton steps and a Krylov subspace of dimension 50. For our experiments we choose $\gamma_{\mathrm{nl}}$ so that the JFNK (nearly) reaches numerical working precision.

\subsection{Parallelization}

For a parallel algorithm, three issues had to be addressed:

(1) scalar product for computing the $L_{2}$-norm

(2) parallelization of the Jacobian times vector operation

(3) parallelization of the preconditioning operation

The MITgcm is MPI-parallelized with domain decomposition [20]. We can use the MITgcm primitives for computing global sums to obtain the scalar product for the $L_{2}$-norm. The parallel Jacobian times vector operation and the preconditioning technique require that all fields are available sufficiently 
far into the computational overlaps. This can be accomplished by one exchange (filling of overlaps, again by MITgcm primitives) for each velocity component before these operations. The remaining parallelization of the preconditioning operation is simplified by using the existing LSR-algorithm in the Picard solver. The convergence of the iterative preconditioning method, and hence of FGMRES linear solver, was greatly improved by introducing a restrictive additive Schwarz method (RASM): in each LSR-iteration a solution is obtained on each sub-domain plus overlap and the global solution is combined by disposing of the overlaps [15]. At the end of each LSRiteration, the overlaps are filled once per velocity component. A so-called parallel Newton-Krylov-Schwarz solver has been described in different contexts [e.g., 31, 32].

\subsection{Vectorization}

The MITgcm dynamic kernel code vectorizes with vector operation ratios of $99 \%$ and higher on an NEC SX-8R vector computer. The only part of the code where the algorithm is modified for better vectorization on vector computers is the LSR-method. This method solves tridiagonal systems with the Thomas algorithm [33] along lines of constant $j$ (or $i$ ) for the $u$ (or $v$ ) components separately. The Thomas algorithm cannot be vectorized so that, for better vector performance, the order of the spatial loops have been exchanged. For example, the Thomas algorithm for the $i$-direction is applied to each component of a vector in $j$ with the effect that the solution for $j-1$ is not available when the $j$-th line is solved; instead the values of the previous LSR-iterate are used (see Figure 1). This turns out to slow down the convergence of the LSR-preconditioner enough to inhibit the convergence of the FGMRES solver in many cases (which in turn affects the convergence of the JFNK solver). As a solution to this problem the vectorized $j$-loops with loop increment one is split into two loops with loop increments of two (a black and a white loop), so that in the second (white) loop the solution at $j$ can be computed with an updated solution of the black loop at $j-1$ and $j+1$. This "zebra" or line-coloring-method [34] improves the convergence of the LSRpreconditioner to the extent that the preconditioned FGMRES solver (and consequently the JFNK solver) regains the convergence that is expected - at the cost of halved vector lengths. The LSR-vector code in the Picard solver also suffers from slower convergence than the scalar code but that is compensated by more iterations to satisfy a convergence criterion, so that the "zebra"-method does not lead to a substantial improvement. 


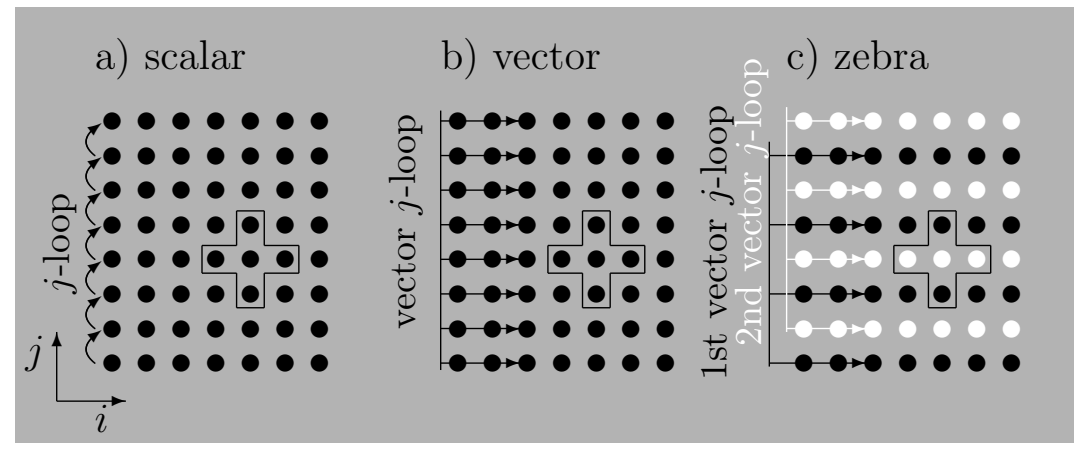

Figure 1: Schematic of LSR-algorithm for the $u$-component of the ice velocities: (a) the scalar code solves a tridiagonal system for each $j$-row sequentially, using known values of row $j-1$ of the current sweep and values of row $j+1$ of the previous sweep for the 5 -point stencil (indicated by the cross); (b) the vector code solves all tridiagonal systems simultaneously, so that only information from previous sweep is available for $j \pm 1$; (c) the "zebra" code solves the black rows simultaneously and then in the second, white sweep the updated information of the black rows $j \pm 1$ can be used.

\subsection{Experiments}

We present simulations of two experimental configurations that demonstrate the overall performance of the JFNK with respect to parallel scaling and vectorization. Comparisons are made with the Picard solver and the

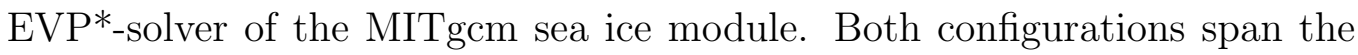
entire Arctic Ocean and in both cases the coupled sea ice-ocean system is driven by prescribed atmospheric reanalysis fields. The ice model is stepped with the same time step as the ocean model and both model components exchange fluxes of heat, fresh water, and momentum interfacial stress at each time level. The two configurations differ in resolution and integration periods. For practical reasons, the atmospheric boundary conditions (i.e., the surface forcing data sets) are very different between these configurations, further excluding any direct comparisons between the simulations. The very interesting comparison of effects of resolution and solvers on climatically relevant properties of the solutions will be described elsewhere.

The first model is used for parallel scaling analysis only. It is based on a simulation with a $4 \mathrm{~km}$ grid spacing on an orthogonal curvilinear grid with 1680 by 1536 grid points [35, 36]. Figure 2 shows the ice distribution and the shear deformation $\dot{\epsilon}_{s}$, both with many small scale structures and linear kinematic features (leads), on Dec-29-2006. For the scaling analysis, the simulation is restarted in winter on Dec-29-2006 with a very small time step size 

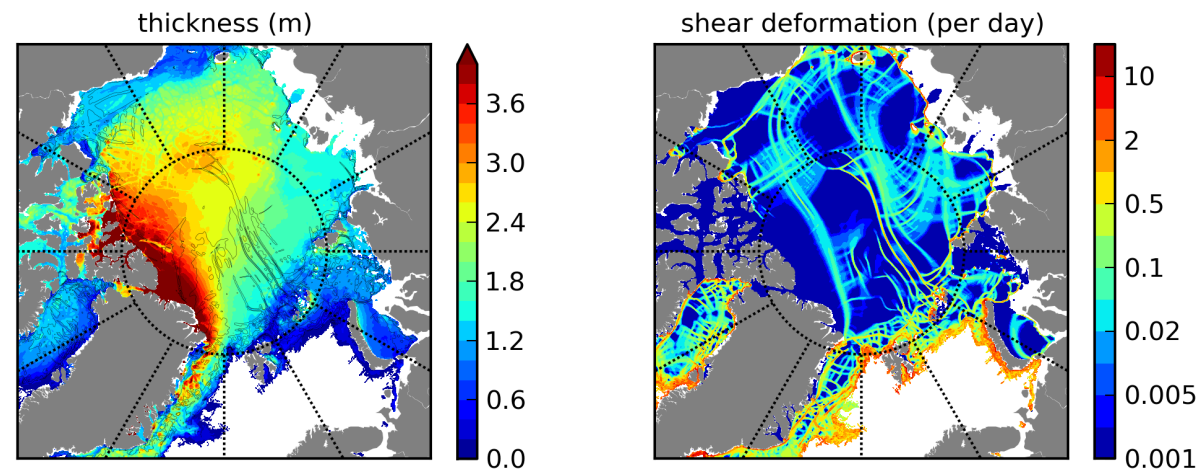

Figure 2: Thickness (m) and concentration (unlabeled contours of 90\%, 95\%, and 99\%) and shear deformation (per day) of the $4 \mathrm{~km}$ resolution model on Dec-29-2006.

of 1 second for a few time steps. For long integrations this time step size is unacceptably small, but here it is necessary because at this resolution the system of equations is even more heterogeneous and ill-conditioned than at lower resolution and the convergence of JFNK (and other solvers) is slower [14]. With larger time steps the number of iterations to convergence (especially when $\gamma_{\mathrm{nl}}$ is small) is different for different numbers of sub-domains (processors) and the scaling results are confounded. All simulations with this configuration are performed either on an SGI UV-100 cluster with Intel ${ }^{\circledR}$ Xeon ${ }^{\circledR}$ CPUs (E7-8837@ $2.67 \mathrm{GHz}$ ) that is available at the computing center of the Alfred Wegener Institute (1-240 CPUs) or on clusters with Intel ${ }^{\circledR}$ Xeon ${ }^{\circledR}$ Gainestown processors (X5570@2.93 GHz) (Nehalem EP) at the North German Supercomputing Alliance (Norddeutscher Verbund für Hochund Höchstleistungsrechnen, HLRN, http://www.hlrn.de) (8-1920 CPUs).

The second model is run on 2 CPUs of an NEC SX-8R vector computer at the computing center of the Alfred Wegener Institute. For these simulations the Arctic Ocean is covered by a rotated quarter-degree grid along longitude and latitude lines so that the equator of the grid passes through the geographical North Pole and the grid spacing is approximately $27 \mathrm{~km}$; the time step size is $20 \mathrm{~min}$. The model is started from rest with zero ice volume on Jan-01-1958 and integrated until Dec-31-2007 with interannually varying reanalysis forcing data of the CORE.v2 data set (http: //data1.gfdl.noaa.gov/nomads/forms/core/COREv2.html). Model grid and configuration are similar to Karcher et al. [37]. Figure 3 shows the 

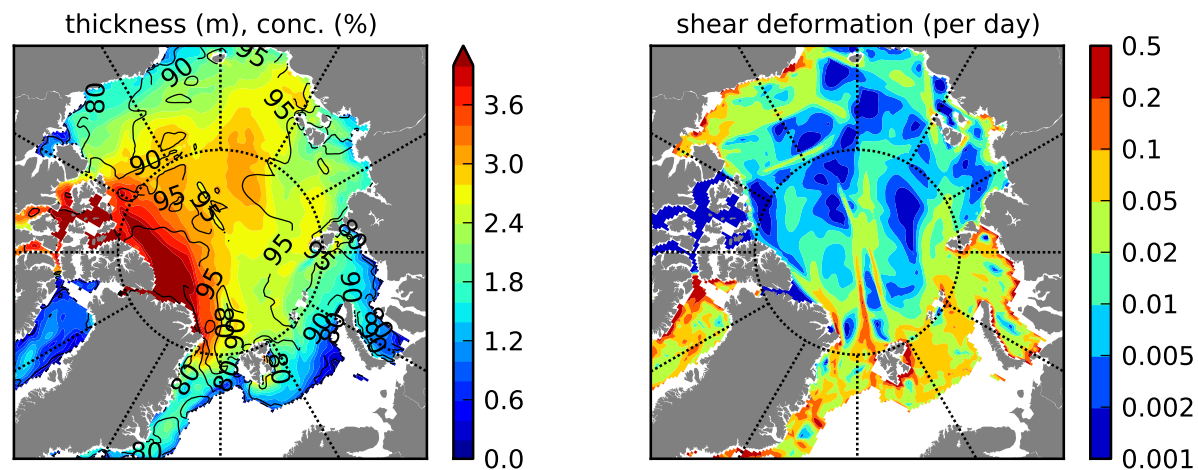

Figure 3: Example ice thickness, concentration (contours), and shear deformation (per day) of the coarse $27 \mathrm{~km}$ resolution model derived from velocity fields on Jun-30-1982.

thickness distribution and the shear deformation of Jun-30-1982 in the simulation. The ice fields are smooth compared to the $4 \mathrm{~km}$-case (Figure 2), but some linear kinematic features also appear in the deformation fields. Note that over the 50 years of simulation $(1,314,864$ time levels) the JFNK-solver failed only 81 times to reach the convergence criterion of $\gamma_{\mathrm{nl}}=10^{-4}$ within 100 iterations corresponding to a failure rate of $0.006 \%$. To our knowledge this is the first successful coupled ice-ocean simulation with a JFNK-solver for the ice-dynamics.

\section{Results}

\subsection{Effect of Jacobian times vector approximation}

For this experiment, the coarse resolution simulation is restarted on Jan01-1966 and the convergence criterion set to $\gamma_{\mathrm{nl}}=10^{-16}$ to force the solver to reach machine precision on the NEC SX-8R. Figure 4 shows that the convergence is a function of $\epsilon$ in (7), but the range of $\epsilon$ for which the finitedifference operation is sufficiently accurate is comfortably large. In practice, full convergence to machine precision will hardly be required so that we can give a range of $\epsilon \in\left[10^{-10}, 10^{-6}\right]$. In this case, using an exact Jacobian by $\mathrm{AD}$ only leads to a very small improvement of one Krylov iteration in the last Newton iteration before machine precision is reached. In all ensuing experiments we use the finite-difference approximation (7) with $\epsilon=10^{-10}$. 

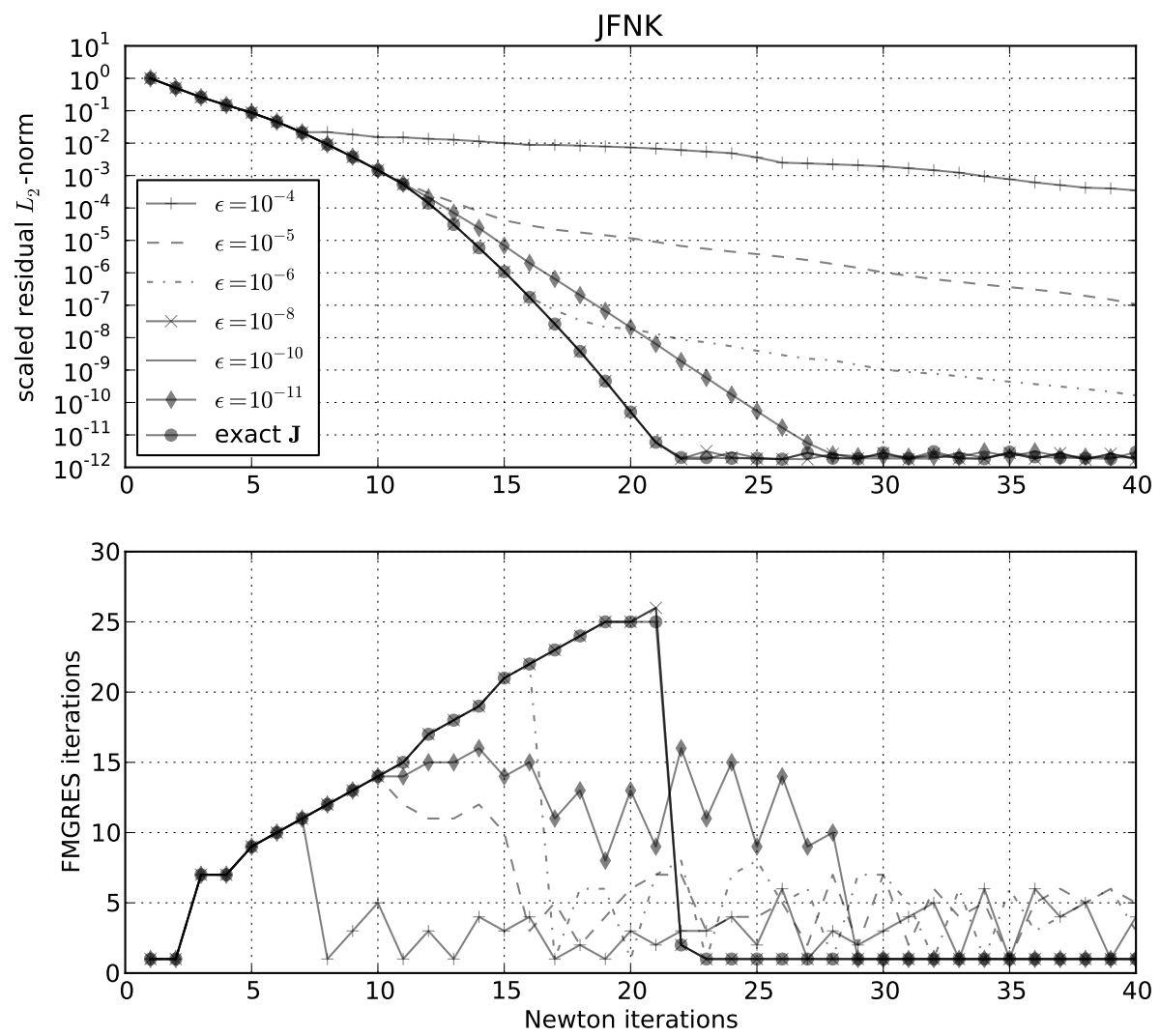

Figure 4: Convergence history of JFNK (top) and total number FGMRES iterations per Newton iteration (bottom) on the NEC SX-8R with different $\epsilon$ for the Jacobian times vector operation. The result with the exact Jacobian time vector operation by $\mathrm{AD}$ is also shown. 


\subsection{Effect of zebra LSR-algorithm}

Figure 5 shows $\left\|\mathbf{F}\left(\mathbf{x}^{k}\right)\right\|$ as a function of the Newton iteration $k$ for three different treatments of the tridiagonal Thomas algorithm in the LSRpreconditioner. The scalar code (Figure 1a) convergences very quickly, but cannot be vectorized so that the time to solution is large. After exchanging the $i$ - and $j$-loops for better vector performance (Figure 1b), the good convergence with the scalar code (solid line) is lost because the convergence rate of the preconditioned FGMRES solver is lower (dashed line). Introducing the "zebra"-method (Figure 1c) recovers the convergence completely (dashdotted line) and maintains the vector performance of the vector code with low extra computational expenses; the code can be vectorized but vector lengths are cut in half compared to the non- "zebra"-code.

\subsection{Effect of RASM on JFNK convergence}

Figure 6 shows that the convergence can be improved with a restricted additive Schwarz Method (RASM) even with an overlap of only 1 grid point. For an overlap of more than 1 the convergence can be still improved in some cases, but not in all (not shown). In general, without writing special exchange primitives for the sea-ice module, the size of the overlap is limited to the total overlap required for general exchange MPI operation (usually not greater than 5) minus the overlap required by the sea-ice dynamics solver (at least 2).

\subsection{Parallel Scaling}

For a credible, unconfounded scaling analysis, the convergence history of the JFNK-solver needs to be independent of the domain decomposition (number of CPUs). For the following analysis the convergence history is exactly the same for all domain decompositions until the 16th Newton iteration; then the models start to deviate from each other because the summations in the scalar product are performed in slightly different order with a different number of sub-domains. As a consequence the number of Newton iterations required to reach the convergence criterion of $\gamma_{\mathrm{nl}}=10^{-10}$ is also different for different domain decompositions. This effect increases with larger time steps. For the present case, the number of Newton and Krylov steps varies moderately between simulations of 4 time steps (121-127 Newton steps and 714-754 Krylov steps), so that we can use the results for a scaling analysis. For comparison, the number of LSR iterations in the Picard solver varies 

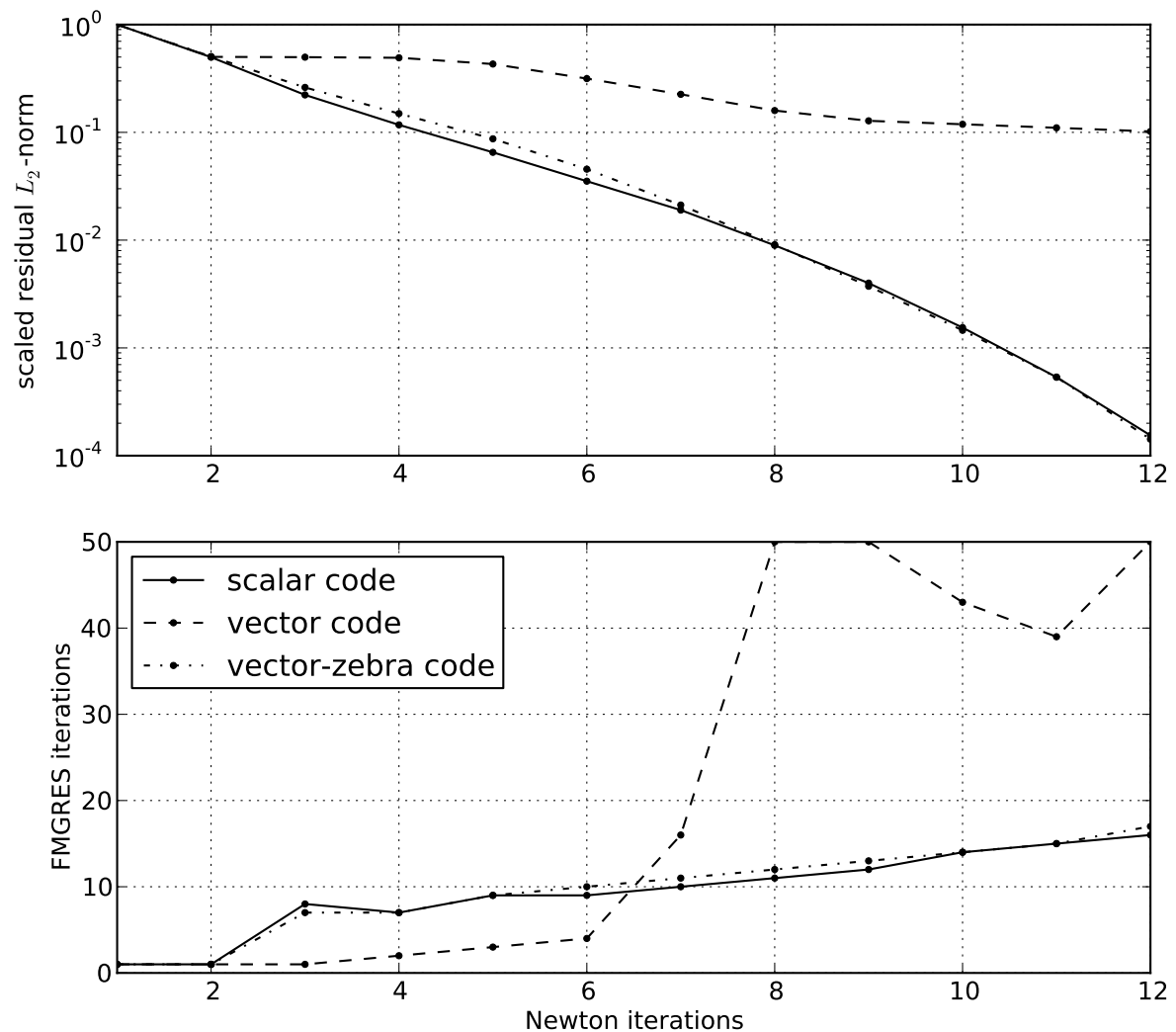

Figure 5: Convergence history of JFNK (top) and total number FGMRES iterations per Newton iteration (bottom) on the NEC SX-8R with different vectorization methods for the tridiagonal Thomas algorithm in LSR. 

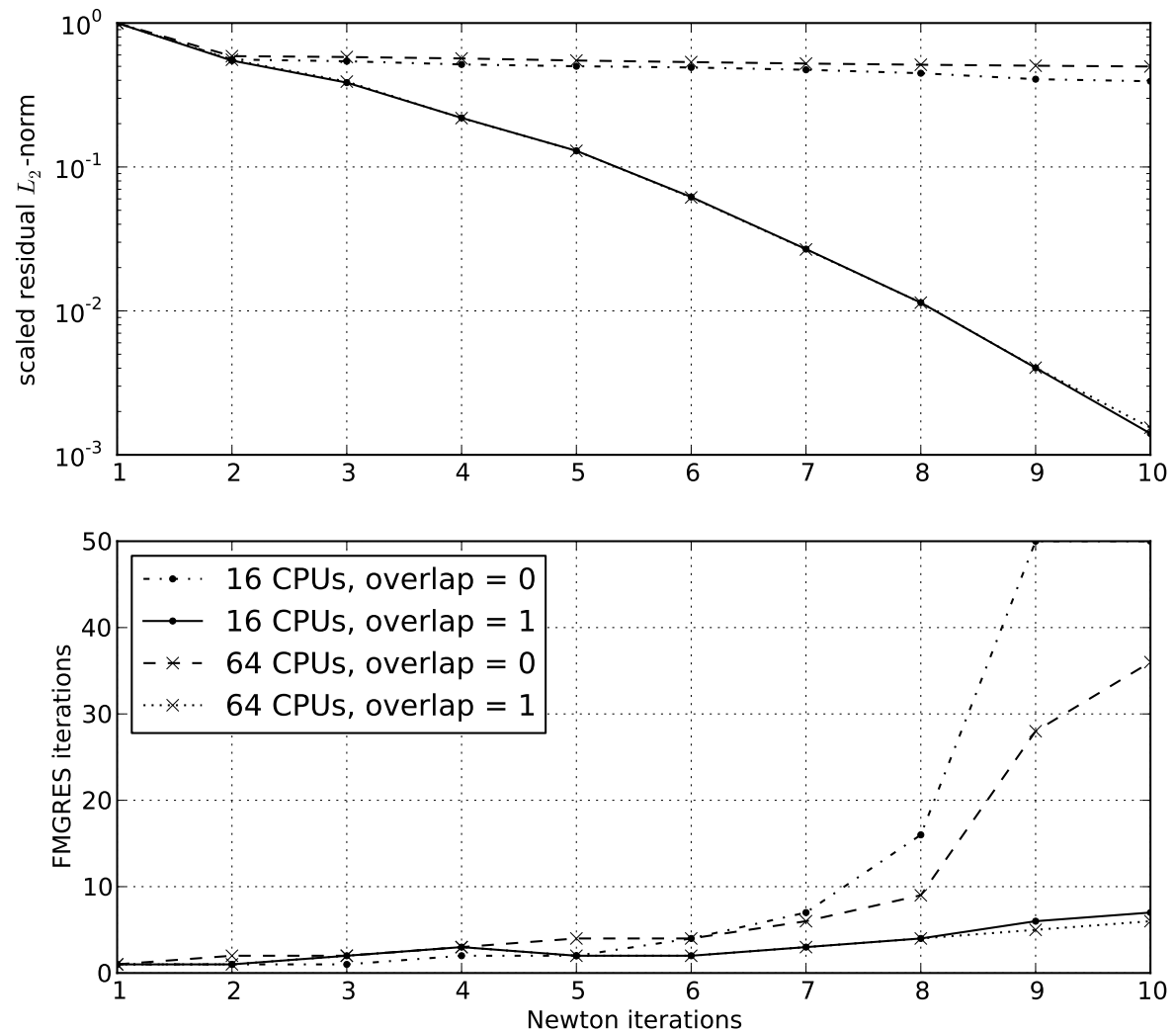

Figure 6: Convergence history of JFNK (top) and total number FGMRES iterations per Newton iteration (bottom) on the SGI-UV100 with and without RASM for 16 and 64 CPUs. "overlap $=0$ " refers to no overlap (no RASM) and "overlap =1" to RASM with an overlap of one. 

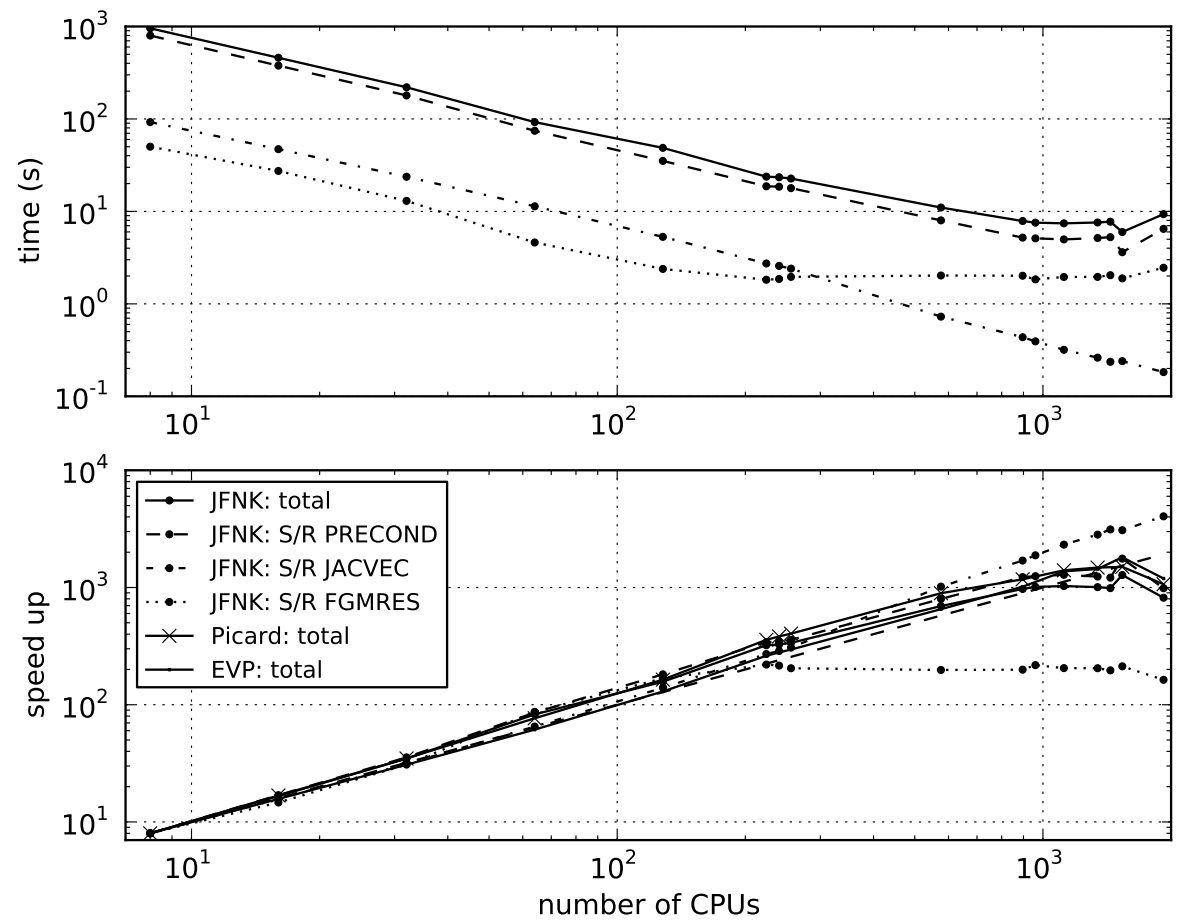

Figure 7: Time for four time steps (top) and relative speed up (bottom) as a function of number of CPUs for the $4 \mathrm{~km}$ resolution configuration on the HLRN computer. The absolute times for the EVP*- and Picard solver are not included.

between 3986 and 4020 in 4 time steps of the same configuration. We confirmed that with a scalar product that preserves the order of summation, this dependence on domain decomposition can be eliminated completely at the cost of very inefficient code.

Figure 7 shows the scaling data obtained from running the models for 4 time steps. For comparison, the results of the Picard solver and the $\mathrm{EVP}^{*}$-solver are included. The EVP*-solver only includes point-to-point communications, but the Picard solver requires point-to-point and collective communications. The JFNK-solver scales linearly as the Picard and the EVP $^{*}$-solver, but reaches a communication overhead earlier (at $10^{3} \mathrm{CPUs}$ ). The routines responsible for this overhead are the many scalar products in the Krylov-method (S/R FGMRES) and the many point-to-point communications within the preconditioning step (S/R PRECOND) (see also [38]). 
Routines that do not require any communication (e.g, S/R JACVEC carries out the Jacobian times vector operation of Eq. (7)) scale linearly to the maximum number of CPUs of 1920, after which the sub-domain size becomes too small to allow linear scaling for the ocean model. Note that both EVP* and Picard solver loose linear scalability above $10^{3} \mathrm{CPUs}$ indicating general limits of the system.

\subsection{Comparison of JFNK to Picard (LSR) and EVP* convergence history}

Figure 8 shows the convergence history of the Picard solver for different termination criteria of the linear LSR-solver and of the JFNK and EVP*solver as a function of scaled linear (inner) iterations. Results are obtained with the $27 \mathrm{~km}$ resolution on the NEC SX-8R. The linear iterations are scaled by the time to solution divided by the total number of linear iterations. For the EVP*-solver, the sub-cycling steps are strictly speaking non-linear iterations, but one such step costs approximately as much as one iteration of a linear solver so that they are only plotted with the linear iterations and not with the non-linear iterations. This pseudo-timing of the EVP*-solver may overestimate its actual cost relative to the other solvers, but in our case the

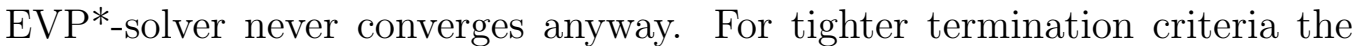
non-linear convergence of the Picard solver improves per non-linear iteration as expected, but also the computational cost increases. Initially, the convergence is actually faster (assuming that each linear iteration takes the same time) for weaker linear convergence criteria. For the case of $\epsilon_{\mathrm{LSR}}=10^{-2}$, the Picard solver even outperforms the JFNK-solver for the first $0.1 \mathrm{~s}$ (approximately 50 linear iterations). Otherwise, the JFNK-solver is more efficient [14], especially after the first couple of non-linear steps. Hence we can confirm that for smaller $\gamma_{\mathrm{nl}}$ the computational advantage of the JFNK-solver over the Picard-solver increases [14]. The EVP*-solver converges faster than the Picard solver for the first $0.5 \mathrm{~s}$ (approximately 250 iterations) and for LSR-convergence criteria $\epsilon_{\mathrm{LSR}}<10^{-4}$, but then it flattens out and oscillates while the Picard solver continues to reduce the residual. For LSR-convergence criteria $\epsilon_{\mathrm{LSR}} \geq 10^{-4}$, the Picard solver always converges faster (see also [10]).

Note that the usual representation of the residual $L_{2}$-norm as a function of non-linear iterations (bottom panel of Figure 8) more clearly shows that the JFNK is always more efficient per non-linear iteration, but this representation is misleading if one is interested in the computational advantage of the JFNK solver. Here the upper panel gives a more realistic representation. 

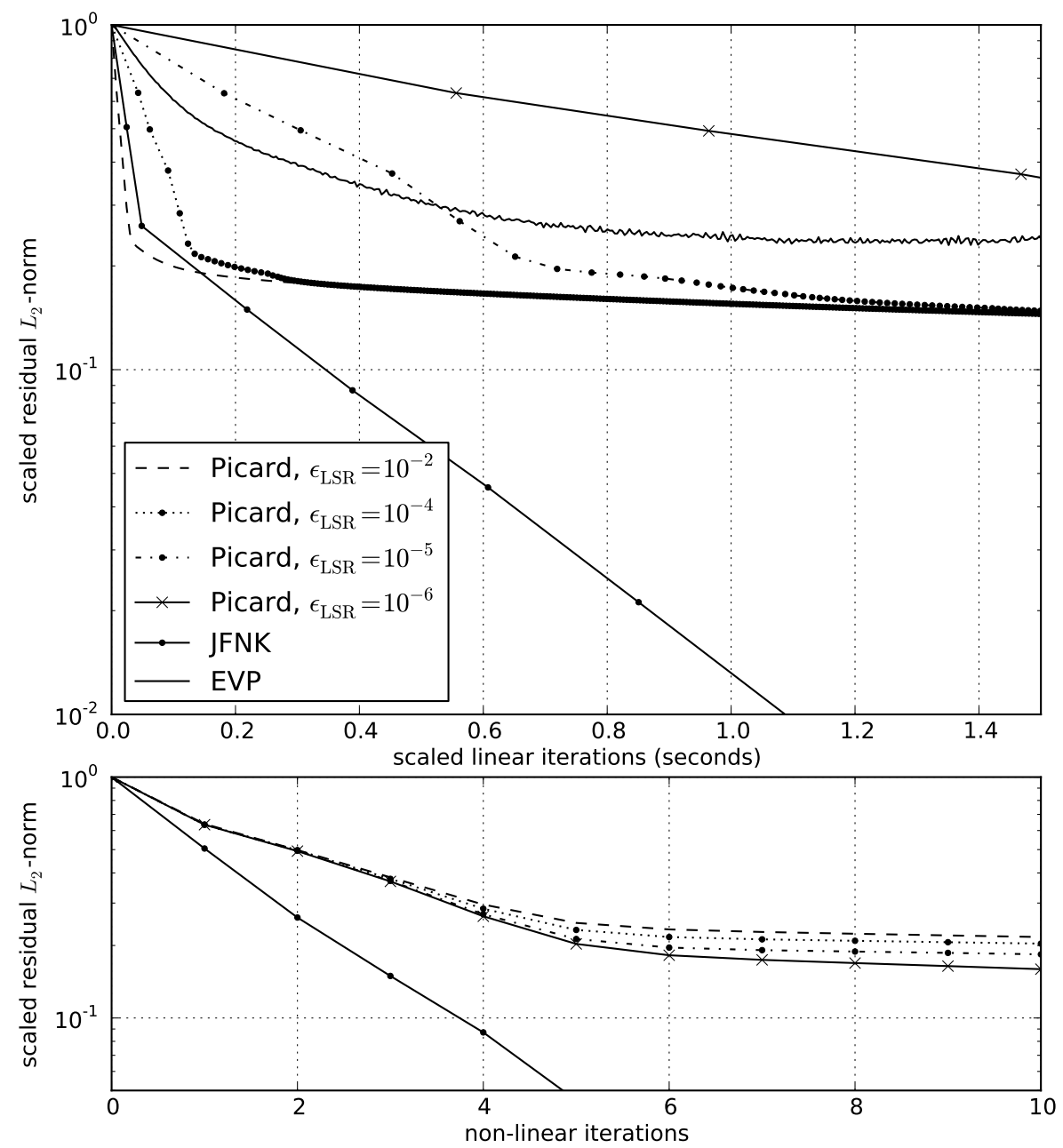

Figure 8: Convergence history of JFNK, EVP*, and Picard solver with different termination criteria for the linear LSR-solver as a function of the number of linear iterations on the NEC SX-8R (top). The number of linear iterations is scaled by the time to solution over total number of linear iteration. The dots and crosses mark the beginning of a new non-linear iteration. The bottom panel shows the residual (scaled by the initial residual) as a function of non-linear iterations. 


\section{Discussion and Conclusions}

Applying the JFNK-method for solving the momentum equations in the sea-ice module of a general circulation model for climate studies requires adaptation and optimization of the method to high performance computer environments. After parallelization and vectorization, the JFNK solver is as successful as the serial version $[14,10]$ in minimizing the $L_{2}$-norm of the residual of the equations. In our experiments the ratio of computational effort (measured in number of iterations of the linear solver) to achieved residual reduction is better for the JFNK-solver than for the traditional Picard-solver and the EVP*-solver. Only for very few linear iterations, a properly tuned Picard-solver can outperform the JFNK-solver. A combination of Picard and JFNK-solver may be an optimal solution [18].

The JFNK-solver runs efficiently on vector computers (here: NEC SX$8 \mathrm{R}$ ), and it scales on massive parallel computers down to a domain size of approximately 50 by 50 grid points (approximately 1000 CPUs in our test). The bottlenecks are a communication overhead in point-to-point exchanges of the preconditioning operation and eventually a communication overhead incurred by many scalar products (collective communication) in the FGMRESsolver. Alternative methods, for example, replacing the Gram-Schmidtorthogonalization in the FGMRES implementation by a Householder-reflection method may alleviate the latter [17], but the former overhead will be felt by all solvers. The flattening of the scaling curves of the Picard- and EVP*solver at the very end of the scaling curve is likely caused by the point-topoint communication overhead.

While adapting the JFNK-solver to parallel or vector architectures is generally straightforward, the preconditioning operator requires more care. This operation is the single most expensive routine in the JFNK-code (Figure 7), because in each FGMRES-iteration it requires (in our case) ten LSR-loops, each with one exchange of the solution vector field, so that an efficient treatment of this part of the code is very important. Further, the convergence of the FGMRES linear solver critically depends on the preconditioning operation and required introducing a restricted additive Schwarz Method (RASM) with an overlap of at least one for parallel applications. For the vector code, the LSR-preconditioner requires a "zebra"-method to ensure good performance of the FGMRES solver. Without the RASM and "zebra" methods, the preconditioned FGMRES sometimes does not converge before the allowed maximum 50 Krylov iterations. These failures of FGMRES then affects the 
nonlinear convergence of the JFNK solver. Furthermore, as our JFNK solver is based on an inexact Newton method, a lower convergence rate of the preconditioned FGMRES solver can also affect the overall nonlinear convergence.

In order to reduce the computational cost of the expensive iterative LSRpreconditioner, a direct (but approximate) procedure, such as a variant of incomplete LU factorization (ILU), could be used. Such a direct method requires only one set of point-to-point communications per FGMRES iteration (instead of ten). Since the factorization itself is difficult to parallelize, the method operates sequentially on each of the sub-domains defined by RASM. There are methods for partial vectorization of ILU [39]. The approximate nature of such a preconditioning operation may require more iterations of FGMRES, and the actual overall performance remains to be demonstrated.

The accuracy of the Jacobian times vector operation was found to be less critical. The exact operation with code obtained with AD slightly reduced the number required iterations to reach work precision compared to forward finite-difference (FD) code with a comfortable range of increments $\epsilon$. With the AD-code the JFNK-solver still took slightly more time (order 2\%), because each Jacobian times vector operation requires two model evaluations, forward model and tangent linear model, while the forward FD code requires only one extra forward model evaluation. The AD-code evaluates forward and tangent-linear model simultaneously, explaining the small overhead.

The current practice in climate modeling of using a Picard solver with a low number of non-linear iterations or using the fast but poorly converging EVP-solver leads to approximate solutions (large residuals) of the sea ice momentum equations. Investing extra computational time with a JFNKsolver - for example, 500 LSR-iterations per time level instead of order 20can reduce this residual by 2 orders of magnitude and more. It has been demonstrated that the differences between sea ice models with more and less accurate solvers can easily reach $2-5 \mathrm{~cm} / \mathrm{s}$ in ice drift velocities and $50 \mathrm{~cm}$ to meters in ice thickness after only one month of integration [9]. These differences are comparable to the differences due to other parameter choices such as the advection scheme for thickness and concentration or the choice of rheology, boundary conditions, or even grid-staggering [12]. We will not speculate to what extent the extra accuracy of a JFNK-solver is required in climate models, but for studying details of sea ice physics and rheology, an accurate solver-technology seems in place to be able to differentiate between numerical artifacts and physical effects. Our implementation of a parallel 
JFNK-solver for sea ice dynamics in an ocean general circulation model is a tool to explore new questions of rheology and sea-ice dynamics in the context of large-scale and computationally challenging simulations that require parallelized codes.

Acknowledgements. We thank Dimitris Menemenlis and An Nguyen for providing the high-resolution configuration to us. Some of the computations were carried out at the North German Supercomputing Alliance (Norddeutscher Verbund für Hoch- und Höchstleistungsrechnen, HLRN).

\section{References}

[1] A. Proshutinsky, Z. Kowalik, Preface to special section on Arctic Ocean Model Intercomparison Project (AOMIP) studies and results, J. Geophys. Res. 112 (2007).

[2] P. Rampal, J. Weiss, C. Dubois, J.-M. Campin, IPCC climate models do not capture Arctic sea ice drift acceleration: Consequences in terms of projected sea ice thinning and decline, J. Geophys. Res. 116 (2011).

[3] W. D. Hibler, III, A dynamic thermodynamic sea ice model, J. Phys. Oceanogr. 9 (1979) 815-846.

[4] A. V. Wilchinsky, D. L. Feltham, Modelling the rheology of sea ice as a collection of diamond-shaped floes, Journal of Non-Newtonian Fluid Mechanics 138 (2006) 22-32.

[5] D. L. Feltham, Sea ice rheology, Ann. Rev. Fluid Mech. 40 (2008) 91-112.

[6] M. Tsamados, D. L. Feltham, A. V. Wilchinsky, Impact of a new anisotropic rheology on simulations of Arctic sea ice, J. Geophys. Res. 118 (2013) 91-107.

[7] K. Wang, C. Wang, Modeling linear kinematic features in pack ice, J. Geophys. Res. 114 (2009).

[8] L. Girard, S. Bouillon, W. Jérôme, D. Amitrano, T. Fichefet, V. Legat, A new modelling framework for sea ice models based on elasto-brittle rheology, Ann. Glaciol. 52 (2011) 123-132. 
[9] J.-F. Lemieux, B. Tremblay, Numerical convergence of viscous-plastic sea ice models, J. Geophys. Res. 114 (2009).

[10] J.-F. Lemieux, D. Knoll, B. Tremblay, D. M. Holland, M. Losch, A comparison of the Jacobian-free Newton-Krylov method and the EVP model for solving the sea ice momentum equation with a viscous-plastic formulation: a serial algorithm study, J. Comp. Phys. 231 (2012) 59265944.

[11] J. Zhang, W. D. Hibler, III, On an efficient numerical method for modeling sea ice dynamics, J. Geophys. Res. 102 (1997) 8691-8702.

[12] M. Losch, D. Menemenlis, J.-M. Campin, P. Heimbach, C. Hill, On the formulation of sea-ice models. Part 1: Effects of different solver implementations and parameterizations, Ocean Modelling 33 (2010) $129-144$.

[13] M. Losch, S. Danilov, On solving the momentum equations of dynamic sea ice models with implicit solvers and the Elastic Viscous-Plastic technique, Ocean Modelling 41 (2012) 42-52.

[14] J.-F. Lemieux, B. Tremblay, J. Sedláček, P. Tupper, S. Thomas, D. Huard, J.-P. Auclair, Improving the numerical convergence of viscous-plastic sea ice models with the Jacobian-free Newton-Krylov method, J. Comp. Phys. 229 (2010) 2840-2852.

[15] X.-C. Cai, M. Sarkis, A restricted additive Schwarz preconditioner for general sparse linear systems, SIAM J. Sci. Comput. 21 (1999) 792-797.

[16] P. D. Hovland, L. C. McInnes, Parallel simulation of compressible flow using automatic differentiation and PETSc, Parallel Computing 27 (2001) 503-519.

[17] W. F. Godoy, X. Liu, Parallel Jacobian-free Newton Krylov solution of the discrete ordinates method with flux limiters for 3D radiative transfer, J. Comp. Phys. 231 (2012) 4257-4278.

[18] C. Paniconi, M. Putti, A comparison of Picard and Newton iteration in the numerical solution of multidimensional variably saturated flow problems, Water Resour. Res. 30 (1994) 3357-3374. 
[19] J. Marshall, A. Adcroft, C. Hill, L. Perelman, C. Heisey, A finite-volume, incompressible Navier Stokes model for studies of the ocean on parallel computers, J. Geophys. Res. 102 (1997) 5753-5766.

[20] MITgcm Group, MITgcm User Manual, Online documentation, MIT/EAPS, Cambridge, MA 02139, USA, 2012. http://mitgcm.org/ public/r2_manual/latest/online_documents.

[21] W. Hundsdorfer, R. A. Trompert, Method of lines and direct discretization: a comparison for linear advection, Applied Numerical Mathematics 13 (1994) 469-490.

[22] P. Roe, Some contributions to the modelling of discontinuous flows, in: B. Engquist, S. Osher, R. Somerville (Eds.), Large-Scale Computations in Fluid Mechanics, volume 22 of Lectures in Applied Mathematics, American Mathematical Society, 1985, pp. 163-193.

[23] C. A. Geiger, W. D. Hibler, III, S. F. Ackley, Large-scale sea ice drift and deformation: Comparison between models and observations in the western Weddell Sea during 1992, J. Geophys. Res. 103 (1998) 2189321913.

[24] J.-F. Lemieux, B. Tremblay, S. Thomas, J. Sedláček, L. A. Mysak, Using the preconditioned Generalized Minimum RESidual (GMRES) method to solve the sea-ice momentum equation, J. Geophys. Res. 113 (2008).

[25] E. C. Hunke, J. K. Dukowicz, The elastic-viscous-plastic sea ice dynamics model in general orthogonal curvilinear coordinates on a sphere incorporation of metric terms, Mon. Weather Rev. 130 (2002) 18471865.

[26] D. Knoll, D. Keyes, Jacobian-free Newton-Krylov methods: a survey of approaches and applications, J. Comp. Phys. 193 (2004) 357-397.

[27] R. Giering, T. Kaminski, Recipes for adjoint code construction, ACM Trans. Math. Softw. 24 (1998) 437-474.

[28] P. Heimbach, C. Hill, R. Giering, An efficient exact adjoint of the parallel MIT general circulation model, generated via automatic differentiation, Future Generation Computer Systems (FGCS) 21 (2005) 1356-1371. 
[29] Y. Saad, A flexible inner-outer preconditioned GMRES method, SIAM J. Sci. Comput. 14 (1993) 461-469.

[30] S. C. Eisenstat, H. F. Walker, Choosing the forcing terms in an inexact Newton method, SIAM J. Sci. Comput. 17 (1996) 16-32.

[31] X.-C. Cai, W. D. Gropp, D. E. Keyes, M. D. Tidriri, Newton-KrylovSchwarz methods in CFD, in: F.-K. Hebeker, R. Rannacher, G. Wittum (Eds.), Proceedings of the International Workshop on the Navier-Stokes Equations, pp. 17-30.

[32] X.-C. Cai, W. D. Gropp, D. E. Keyes, R. G. Melvin, D. P. Young, Parallel Newton-Krylov-Schwarz algorithms for the transonic full potential equation, SIAM J. Sci. Comput. 19 (1998) 246-265.

[33] L. H. Thomas, Elliptic problems in linear differential equations over a network, Watson Sci. Comput. Lab Report, Columbia University, New York, 1949.

[34] I. S. Duff, G. A. Meurant, The effect of ordering on preconditioned conjugate gradients, BIT Numerical Mathematics 29 (1989) 635-657.

[35] A. T. Nguyen, R. Kwok, D. Menemenlis, Source and pathway of the Western Arctic upper halocline in a data-constrained coupled ocean and sea ice model, J. Phys. Oceanogr. 43 (2012) 80-823.

[36] E. Rignot, I. Fenty, D. Menemenlis, Y. Xu, Spreading of warm ocean waters around Greenland as a possible cause for glacier acceleration, Ann. Glaciol. 53 (2012) 257-266.

[37] M. Karcher, A. Beszczynska-Möller, F. Kauker, R. Gerdes, S. Heyen, B. Rudels, U. Schauer, Arctic ocean warming and its consequences for the Denmark Strait overflow, J. Geophys. Res. 116 (2011).

[38] C. Hill, D. Menemenlis, B. Ciotti, C. Henze, Investigating solution convergence in a global ocean model using a 2048-processor cluster of distributed shared memory machines, Scientific Programming 12 (2007) $107-115$.

[39] J. J. F. M. Schlichting, H. A. van der Vorst, Solving 3D block bidiagonal linear systems on vector computers, Journal of Computational and Applied Mathematics 27 (1989) 323-330. 